\title{
Neutron correlations in the decay of the first excited state of ${ }^{11} \mathrm{Li}$
}

J. K. Smitha,b,1,*, T. Baumann ${ }^{\mathrm{a}}$, D. Bazin ${ }^{\mathrm{a}}$, J. Brown ${ }^{\mathrm{c}}$, P. A. DeYoung ${ }^{\mathrm{d}}$, N. Frank ${ }^{\mathrm{e}}$, M. D. Jones ${ }^{\mathrm{a}, \mathrm{b}, 2}$, Z. Kohley ${ }^{\mathrm{a}, \mathrm{f}}$, B. Luther ${ }^{\mathrm{g}}$, B. Marks ${ }^{\mathrm{d}}$, A. Spyrou ${ }^{\mathrm{a}, \mathrm{b}}$, S. L. Stephenson ${ }^{\mathrm{h}}$, M. Thoennessen ${ }^{\mathrm{a}, \mathrm{b}}, \mathrm{A}$. Volya ${ }^{\mathrm{i}}$

\author{
${ }^{a}$ National Superconducting Cyclotron Laboratory, East Lansing, MI 48824 \\ ${ }^{b}$ Department of Physics, Michigan State University, East Lansing, MI 48824 \\ ${ }^{c}$ Department of Physics, Wabash College, Crawfordsville, IN 47933 \\ ${ }^{d}$ Department of Physics, Hope College, Holland, MI 49422 \\ ${ }^{e}$ Department of Physics and Astronomy, Augustana College, Rock Island, IL 61201 \\ ${ }^{f}$ Department of Chemistry, Michigan State University, East Lansing, MI 48824 \\ ${ }^{g}$ Department of Physics, Concordia College, Moorhead, MN 56562 \\ ${ }^{h}$ Department of Physics, Gettysburg College, Gettysburg, PA 17325 \\ ${ }^{i}$ Department of Physics, Florida State University, Tallahassee, FL 32306
}

\begin{abstract}
The decay of unbound excited ${ }^{11} \mathrm{Li}$ was measured after being populated by a two-proton removal from a ${ }^{13} \mathrm{~B}$ beam at $71 \mathrm{MeV} /$ nucleon. Decay energy spectra and Jacobi plots were obtained from measurements of the momentum vectors of the ${ }^{9} \mathrm{Li}$ fragment and neutrons. A resonance at an excitation energy of $\sim 1.2 \mathrm{MeV}$ was observed. The kinematics of the decay are equally well fit by a simple dineutron-like model or a phase-space model that includes final state interactions. A sequential decay model can be excluded.
\end{abstract}

Keywords: neutron spectroscopy, exotic nuclei, halo nuclei, three-body correlations

\section{Introduction}

The emergence of simple behavior from complex many-body systems is central to nuclear physics. In particular, a class of nuclei called halo nuclei are usually simplified as a cluster-like few-body problem of a core and halo nucleon(s). The internal structure of the core and the dynamics of the halo nucleons have been studied extensively (see for example [1-3]).

For the two-neutron halo in ${ }^{11} \mathrm{Li}$, the question of the correlation between the two valence neutrons in the ground state has attracted much attention. There is agreement on the average distance between the two neutrons in the ground state $[4,5]$, but beyond that there have been conflicting results. Between 1992 and 1995, data from three different

\footnotetext{
${ }^{*}$ Corresponding author

Email address: jsmith@triumf.ca (J. K. Smith)

${ }^{1}$ Current address: TRIUMF, Vancouver, BC V6T 2A3, Canada

${ }^{2}$ Current address: LBNL, Berkeley, CA 94720

Preprint submitted to Nuclear Physics A
}

May 28, 2016 
experiments were published [6-9]. Each experiment claimed different decay models for the $2 n$ decay: sequential [6], phase-space [7, 8], and dineutron [9]. Since then, one experiment has ruled out dineutron decay [10], one experiment has claimed evidence for sequential decay [11], and two others have shown evidence for correlation between the two neutrons $[12,13]$. Many of these experiments utilized Coulomb excitation into the continuum for their work $[7-10,13]$ since the ground state of ${ }^{11} \mathrm{Li}$ is bound $\left(S_{n}=369.15 \pm 0.65 \mathrm{keV}\right.$ [14]) and there are no bound excited states. Though the energies observed were in the continuum, the neutron-neutron correlations observed were those of the ground-state configuration, since the $E 1$ operator only acts on the center-of-mass coordinate of the neutron-neutron pair, not the relative coordinate [9]. This distinction does not, however, resolve the conflict.

Here, we report on the first correlation measurement of the decay of the first excited state in ${ }^{11} \mathrm{Li}$ populated with a two-proton removal reaction from ${ }^{13} \mathrm{~B}$. We also compare this measurement to a handful of simple models for this complex system. The first unbound excited state has been previously measured at approximately $1.2 \mathrm{MeV}[10-$ $12,15-18]$. Our results are in agreement with the previous measurements and expand the study of this state using two-nucleon correlation techniques [19-25].

\section{Experimental set-up and results}

The experiment was performed at the National Superconducting Cyclotron Laboratory. A ${ }^{13} \mathrm{~B}$ secondary beam was provided by the Coupled Cyclotron Facility [26] and the A1900 Fragment Separator [27]. The secondary beam, at $71 \mathrm{MeV} / \mathrm{u}$, reacted in a beryllium target and produced ${ }^{11} \mathrm{Li}$ by two-proton removal. The ${ }^{11} \mathrm{Li}$ nuclei in unbound excited states immediately decayed to ${ }^{9} \mathrm{Li}$ and two neutrons. Charged fragments were detected with a series of charged particle detectors after being deflected by the Sweeper high-field dipole magnet [28]. The neutrons were detected via their interactions in MoNALISA, an array of plastic scintillator bars [29]. The positions and angles measured in the charged particle detectors were used to track the particles back through the Sweeper magnet and calculate their momentum vectors leaving the target. For this experiment, MoNA-LISA's 288 bars were arranged in 3 groups of nine walls, four walls, and five walls. The nine- and four-wall groups were placed at zero degrees approximately 9 and $7.8 \mathrm{~m}$ from the target, respectively. The 5 -wall group was placed $6.8 \mathrm{~m}$ from the target at $23^{\circ}$. The momentum vectors of the three decay products were used to calculate the decay energy of the unbound ${ }^{11} \mathrm{Li}$ system with the invariant mass method. More details are available in Refs. [30, 31].

The four decay-energy spectra measured in this experiment are shown in Figure 1. The top row shows two-body decay energy spectra $\left({ }^{9} \mathrm{Li}+n\right)$, while the bottom row shows three-body decay energy spectra $\left({ }^{9} \mathrm{Li}+n+n\right)$. The spectra in panels (a) and (c) are both ungated, while the spectra in panels (b) and (d) have been gated to enhance different physics events present in the data. Events that contain a ${ }^{9} \mathrm{Li}$ daughter fragment and one or more neutron interactions could come from either $2 p$ knockout to ${ }^{11} \mathrm{Li}$ and subsequent $2 n$ decay or a $2 p 1 n$ knockout directly to ${ }^{10} \mathrm{Li}$ and subsequent $1 n$ decay. Figure $1(\mathrm{~b})$ shows the two-body spectrum for events that have only a single interaction in MoNALISA. This enhances the $1 n$ decay signal created by direct population of ${ }^{10} \mathrm{Li}$ via $2 p 1 n$ knockout from the ${ }^{13} \mathrm{~B}$ beam. 

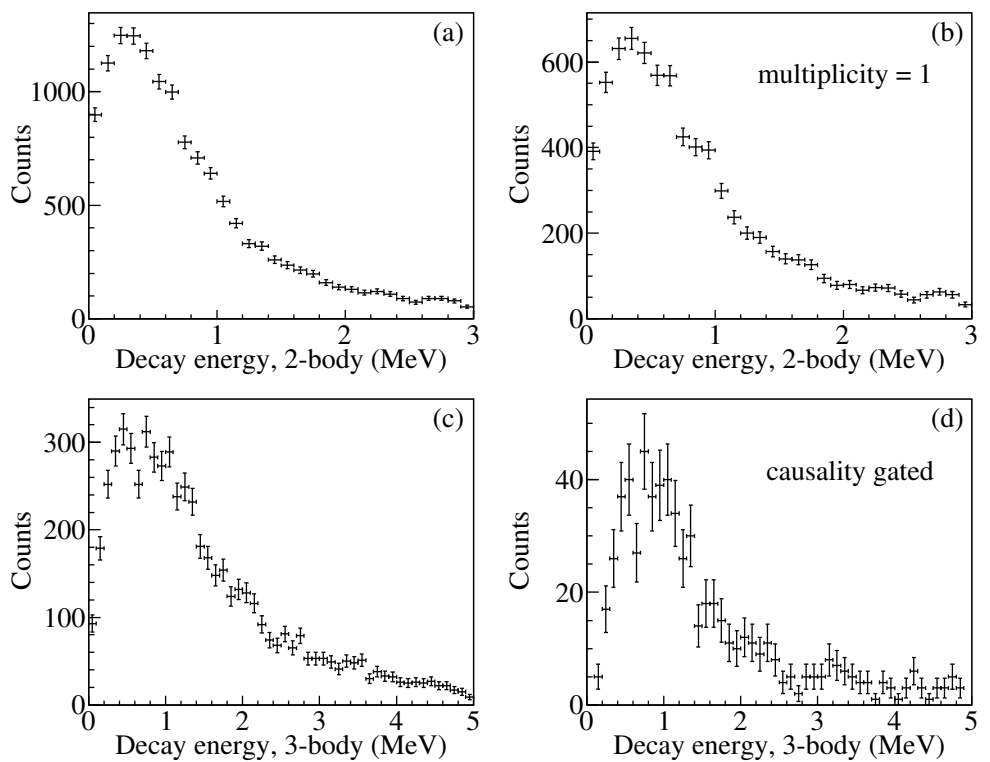

Figure 1: Two- (top row) and three-body (bottom row) decay energy spectra $\left({ }^{9} \mathrm{Li}+n\right.$ and ${ }^{9} \mathrm{Li}+n+$ $n)$. Panel (a) shows the ungated two-body decay energy spectrum while panel (b) shows the twobody decay energy spectrum for events with only a single interaction in MoNA-LISA. Panel (c) shows the ungated three-body decay energy spectrum, while panel (d) shows the causality-gated three-body spectrum (details in text).

The causality gate that has been applied in Figure $1(\mathrm{~d})$ is designed to enhance the $2 n$ signal from decay of ${ }^{11} \mathrm{Li}$ and is critical to the analysis of two-neutron decay data. It enhances the signal from real two-neutron events by reducing the contribution from many single-neutron events (where a single neutron is scattered and detected multiple times in the detector array). The single-neutron events could be caused by direct population and subsequent decay of ${ }^{10} \mathrm{Li}$ or from the interaction of only one of the two neutrons emitted from a ${ }^{11} \mathrm{Li}$ decay. Unfortunately, distinguishing real two-neutron events from single-neutron events is not possible in all cases on an event-by-event basis.

Signals from real two neutron events can be enhanced relative to single-neutron events by applying gates based on the kinematics of the events. For each event, the velocity of an assumed imaginary neutron scattered between the first and second interaction points $\left(v_{12}\right)$ is calculated and compared to the velocity of the neutron originating in the target and traveling to the first interaction point $\left(v_{01}\right)$. An event with a scattered single neutron detected multiple times would have $v_{12} \leq v_{01}$. The first causality gate applied required that $v_{12}>v_{01}$. The second causality gate required the two interaction points be separated by at least $50 \mathrm{~cm}$. This eliminated detector-to-detector scattering that passed the first cut due to position and time resolutions. Similar gates have been used in previous analyses of two-neutron decays [13, 22, 23, 32, 33].

After these cuts, the data show a peak at a decay energy of approximately 800 $\mathrm{keV}$ (Figure $1(\mathrm{~d})$ ), corresponding to an excitation energy in ${ }^{11} \mathrm{Li}$ of $\sim 1.2 \mathrm{MeV}$ which is consistent with the previous measurements of Refs. [10-12, 15-18]. 


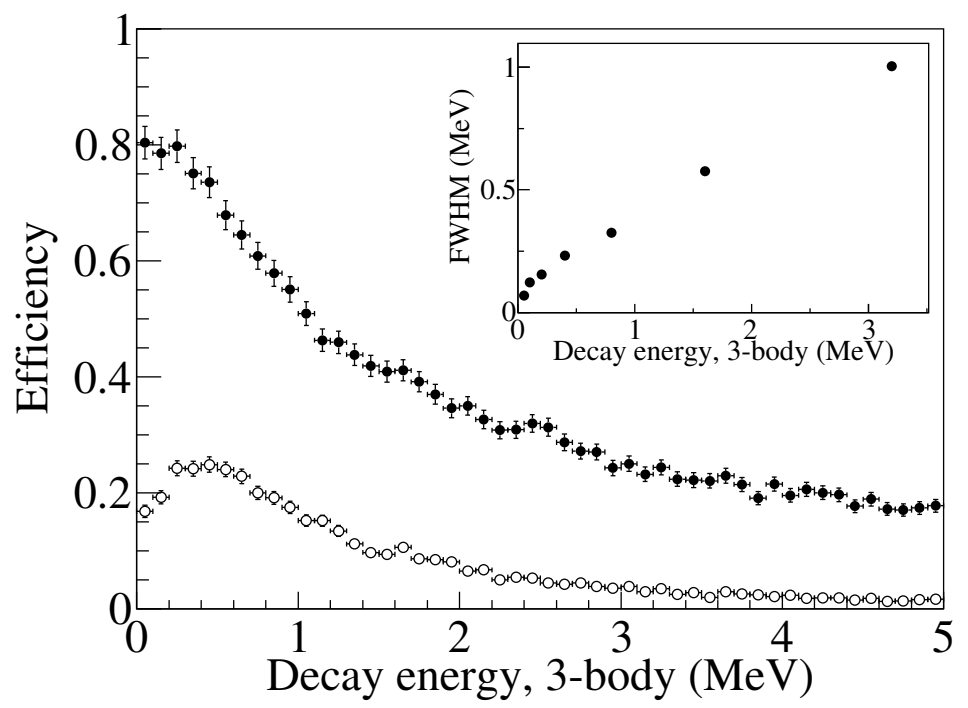

Figure 2: The detection efficiency of MoNA-LISA as a function of three-body decay energy. Solid points show the ungated detection efficiency and open points represent the causality-gated efficiency. Binning has been chosen to be the same as the experimental spectra. Inset plot shows the full-width at halfmaximum (FWHM) experimental resolution after applying a causality gate as a function of three-body decay energy.

\section{Simulation}

In order to compare different theoretical models with the data, the results of the calculations have to be folded with the response function of the experimental setup. A Monte Carlo simulation [34] was used to compare the data with several different decay models. The simulation included a description of the incoming beam profile, the knockout reaction kinematics, the trajectories of the charged fragments in the magnet, and the detector acceptances and resolutions. The interactions of the neutrons with MoNALISA and the subsequent detector response were simulated using GEANT4 [35, 36] and MENATE_R [34] with the cross-section corrections noted in Ref. [25]. The efficiency and full-width at half-maximum (FWHM) resolution of the causality-gated three-body decay energy spectrum are shown in Figure 2. In particular, the FWHM resolution of MoNALISA for a $2 n$ decay of $800 \mathrm{keV}$ energy and with causality cuts applied is calculated by the simulation as $325 \mathrm{keV}$ (Figure 2, inset). A $1 n$ efficiency plot has been previously published in Figure 2a of Ref. [23].

Given the importance of neutron scattering to this experiment, the Monte Carlo simulation was first tested to verify its reproduction of neutron scattering behavior in a one-neutron decay. Specifically, we used a $1 p$ knockout to unbound ${ }^{12} \mathrm{Be}$, followed by a $1 n$ decay to ${ }^{11} \mathrm{Be}$, published previously in Ref. [30]. Figure 3 shows four decay energy spectra: two for the ${ }^{11} \mathrm{Be}+n$ system (top row) and two for the ${ }^{11} \mathrm{Be}+n+n$ system (bottom row). As in Figure 1, the left column is ungated, while panel (b) shows a two-body decay energy spectrum gated on events with a single interaction in MoNA-LISA and panel (d) shows the three-body decay energy spectrum with causality cuts applied. The events in 

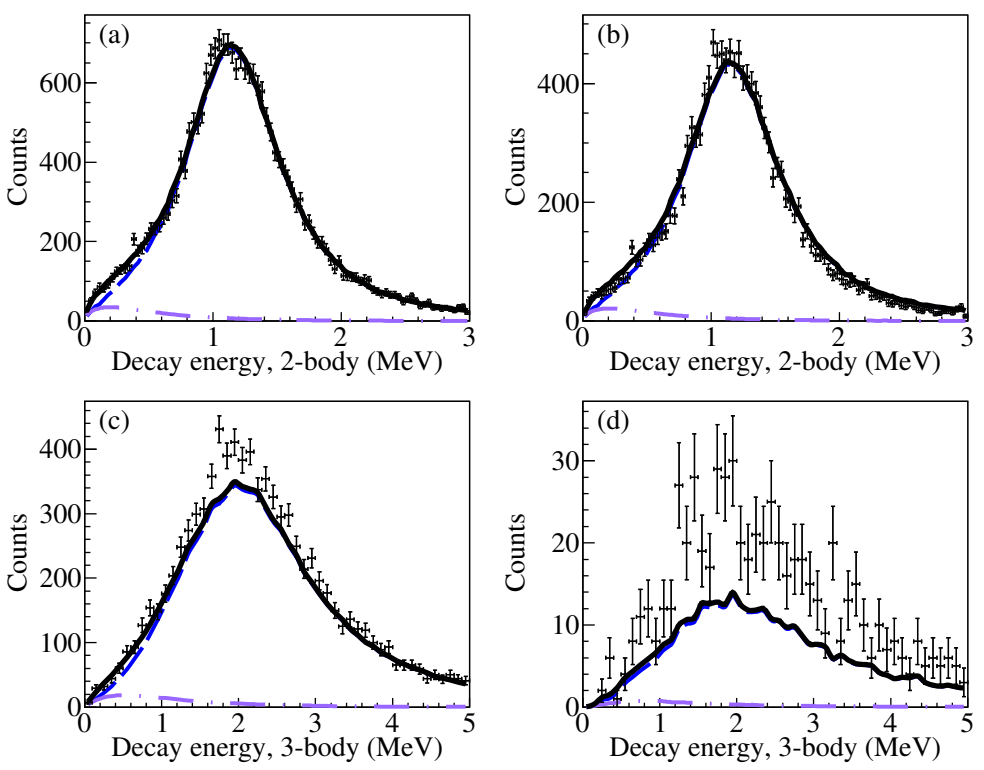

Figure 3: Decay energy spectra in coincidence with an ${ }^{11}$ Be daughter fragment were used to verify the simulation of neutron scattering. Panel (a) shows the two-body decay energy spectrum $\left({ }^{11} \mathrm{Be}+n\right)$, panel (b) shows the same spectrum gated on events with a single interaction in MoNA-LISA. Panel (c) shows the three-body decay energy spectrum $\left({ }^{11} \mathrm{Be}+n+n\right)$ and panel (d) shows the same spectrum with a causality cut. The dashed blue line shows a single resonance and the dot-dashed purple line shows a Maxwellian distribution as used in Ref. [30] and as discussed in the text. The black solid line shows the sum of the two simulations. 
panels (c) and (d) are expected to be largely due to scattered single neutrons from the $1 n$ decay and reproduction of these data by the simulation would indicate that the neutron scattering is being modeled appropriately.

The solid black line in Figure 3 is the sum of two ${ }^{11} \mathrm{Be}+n$ simulations: one with a resonance at $1243 \mathrm{keV}$ (dashed blue line) and one simulating a small background with a Maxwellian distribution. This pair of simulations was fit to only the ungated twobody decay energy spectrum (panel (a)). The simulation reproduces well both the gated two-body decay energy spectrum (panel (b)) and the ungated three-body decay energy spectrum (panel (c)). It fails by a factor of two, however, to reproduce the magnitude of events in the causality-gated three-body spectrum (panel $(d)$ ). This seems to indicate an underprediction of the single neutron scattering cross talk. Although we expect the majority of the events with two neutron interactions to be caused by a single neutron scattering, there could be a small contribution from a nucleon-exchange reaction that would populate ${ }^{13} \mathrm{Be}$ and then decay via the emission of two neutrons. Marks, et al. found that such a reaction occurred with a cross-section one order of magnitude smaller than one-proton knockout in this region [37]. Assuming that this reaction was also the cause of the small background necessary for fitting the ungated two-body decay energy spectrum, we used the simulation reported in Ref. [37] to replace the Maxwellian background distribution and fit the data once again (Figure 4). This simulation not only reproduces the first three decay energy spectra but gives a noticeably better description of the causality-gated three-body decay energy spectrum. The relative cross-section for nucleon exchange was an order of magnitude smaller than the $1 p$ knockout, consistent with Ref. [37]. Based on this test case, we estimate that our simulation of single neutron scattering cross-talk is accurate within $33 \%$.

We then proceeded with the fits of the present ${ }^{11} \mathrm{Li}$ data. The ${ }^{11} \mathrm{Li}$ decay was simulated with five different decay paths:

1. Sequential decay through the ${ }^{10} \mathrm{Li} p$-wave resonance approximately $500 \mathrm{keV}$ above the ${ }^{10} \mathrm{Li}$ ground state (Sequential, $\ell=1$ )

2. Sequential decay through the broad, $s$-wave ${ }^{10} \mathrm{Li}$ ground state (Sequential, $\ell=0$ )

3. Direct decay to ${ }^{9} \mathrm{Li}$ via phase space decay (Phase space)

4. Direct decay to ${ }^{9} \mathrm{Li}$ via phase space decay with a final state interaction (Phase space + FSI)

5. Direct decay to ${ }^{9} \mathrm{Li}$ via dineutron-like decay (Dineutron)

A simplified level scheme of the simulated decay paths is shown in Figure 5.

All models based their total three-body decay energy distributions on an energydependent Breit-Wigner lineshape. The sequential decays [31, 38] were modeled following the description of Ref. [38] as two sequential single-neutron emissions. The phase-space decay was modeled following the description of Ref. [39] as a simultaneous emission of two-neutrons exhibiting three-body kinematics. The phase space + final state interaction model followed the prescription of Marqués et al. [4, 40]. In this model, the interaction between the two neutrons was parametrized based on the average distance between the two neutrons within the nucleus (the source size). The source size of ${ }^{11} \mathrm{Li}$ used in this work was chosen to be the same as in the original paper $\left(r_{\mathrm{nn}}^{\mathrm{rms}}=6.6 \mathrm{fm}\right)$ [4]. The dineutron decay was modeled as an emission of a dineutron and its subsequent decay following the description of Refs. [23, 31] and utilizing the measured dineutron scattering length of $-18.7 \mathrm{fm}[41]$ to characterize the decay of the dineutron. The ${ }^{11} \mathrm{Li}$ resonance energy and 

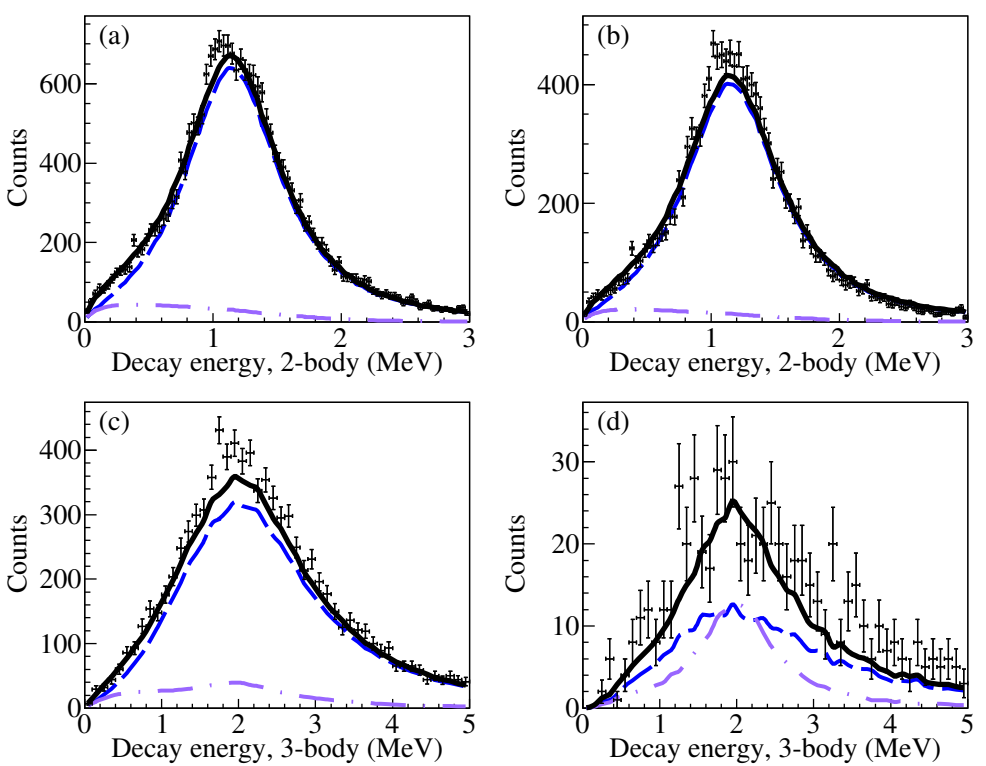

Figure 4: Decay energy spectra in coincidence with an ${ }^{11}$ Be daughter fragment were used to verify the simulation of neutron scattering. Replacing the Maxwellian distribution shown in Figure 3 with a two-neutron decay resulting from a nucleon-exchange reaction [37] improves the reproduction of the three-body decay energy spectrum with causality cuts (d). The data shown are the same as Figure 3, but here, the dashed blue line shows a single resonance and the dot-dashed purple line shows a two-neutron decay distribution resulting from a nucleon-exchange reaction as used in Ref. [37] and as discussed in the text. The black line shows the sum of the two distributions. 


\begin{tabular}{ccccccc}
\hline \hline & \multicolumn{3}{c}{ This work } & \multicolumn{3}{c}{ Literature value } \\
& $E(\mathrm{keV})$ & $\Gamma(\mathrm{keV})$ & FWHM $(\mathrm{keV})$ & $E(\mathrm{keV})$ & FWHM $(\mathrm{keV})$ & Ref. \\
\hline${ }^{10} \mathrm{Li}$ g.s. & 25 & - & - & $27 \pm 13$ & - & {$[42]$} \\
${ }^{10} \mathrm{Li}^{*}$ & 530 & 600 & 310 & $530 \pm 60$ & $350 \pm 80$ & {$[43]$} \\
${ }^{10} \mathrm{Li}^{*}$ & 770 & 1200 & 600 & $770 \pm 240$ & - & {$[44]$} \\
${ }^{10} \mathrm{Li}^{*}$ & 2350 & 1200 & 1100 & $2350 \pm 100$ & $1200 \pm 400$ & {$[43]$} \\
\hline \hline
\end{tabular}

Table 1: ${ }^{10} \mathrm{Li}$ resonance energy and physical width $(\Gamma)$ values used in the simulations compared with the measured full-width-half-maximum (FWHM) of each input distribution and literature values. Here, $\Gamma$ refers to the intrinsic width of the state included in the calculation of the decay energy curve, while FWHM is a measurement of the width of the calculated input decay energy curve, before resolutions and acceptances are applied.

total decay width were free parameters for all five $2 n$ decay paths. The sequential decay paths also allowed as free parameters the energy and width of the intermediate state.

The possibility for a direct population of ${ }^{10} \mathrm{Li}$ was also taken into account. In addition to the above mentioned ground state and excited state, the direct population of two higher lying states (at decay energies of $\sim 1 \mathrm{MeV}$ and $2.35 \mathrm{MeV}$ ) was included in order to describe the high-energy tails of the spectra.

The simulations were fit simultaneously to the four different decay energy spectra presented in Figure 1. Each fit included one $2 n$ decay path and all $1 n$ decay paths. The resonance parameters for each decay, as well as the relative intensities of each decay path, were left as free parameters in each fit.

Initial fits showed that the minimized ${ }^{11} \mathrm{Li}$ resonance parameters were independent of the ${ }^{10} \mathrm{Li}$ resonance parameters. The ${ }^{10} \mathrm{Li}$ parameters were either minimized and consistent with previous literature values or insensitive. For the final minimization of the ${ }^{11} \mathrm{Li}$ energy and width parameters, the ${ }^{10} \mathrm{Li}$ parameters were fixed at the literature values shown in Table 1 . In the cases of the states at 530 and $2350 \mathrm{keV}$, the physical widths were chosen so that the FWHM values would be consistent. Ref. [43] used an energy-independent Breit-Wigner, where the FWHM is the same as the reported physical width. Here we use an energy-dependent Breit-Wigner, whose FWHM is narrower than the physical width $\Gamma$, due to the addition of a penetrability factor $[31,45]$. For the width of the resonance at $770 \mathrm{keV}$ that does not have a literature value, we chose $\Gamma=1200 \mathrm{keV}$, which corresponds to a FWHM of $\sim 600 \mathrm{keV}$.

\section{Results and Discussion}

The fit results for the different decay paths of ${ }^{11} \mathrm{Li}$ are shown in Figures 6 and 7 . Each of the colored lines indicate the best total fit for that decay path, with the single $2 n$ and multiple $1 n$ components summed. All simulated $2 n$ decay paths describe the decay energy spectra equally well. The best-fit ${ }^{11} \mathrm{Li}$ decay energies and widths are shown in Table 2 . The best fit energies vary between 690 and $930 \mathrm{keV}$ (phase space + FSI and dineutron, respectively), but are not necessarily consistent with each other. To pin down the central energy value, it is crucial to identify the appropriate decay model. For the widths, only lower limits could be determined because the FWHM of the energy-dependent BreitWigner used in these simulations saturates at large physical width $(\Gamma[31])$. Despite this 


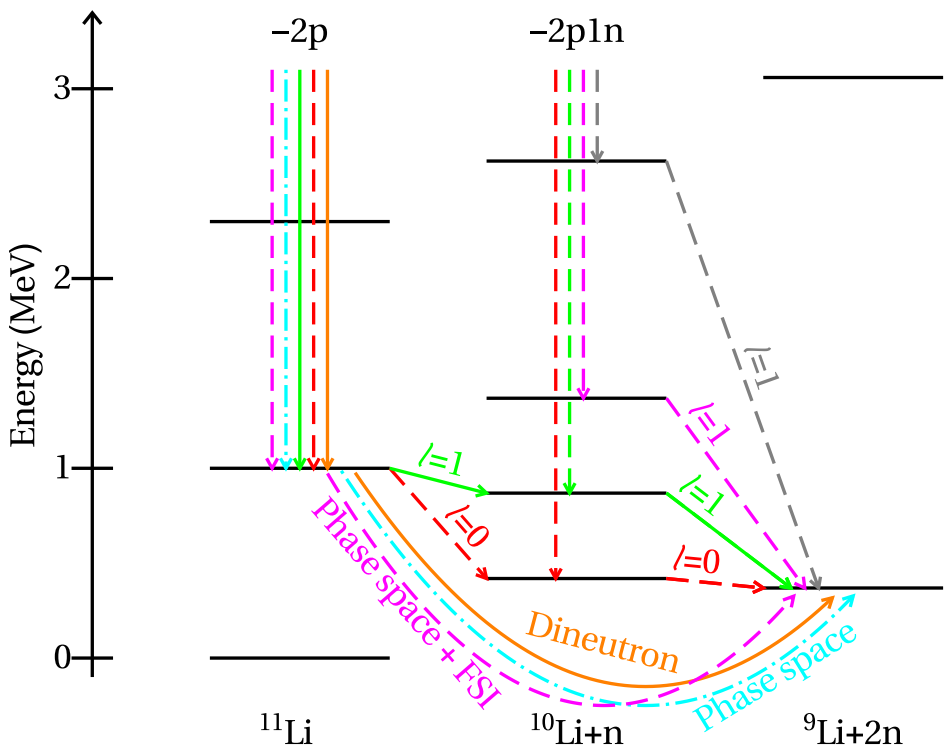

Figure 5: Simplified level scheme for the population and decay of ${ }^{11} \mathrm{Li}$ and ${ }^{10} \mathrm{Li}$ showing the decay paths used in the simulations.
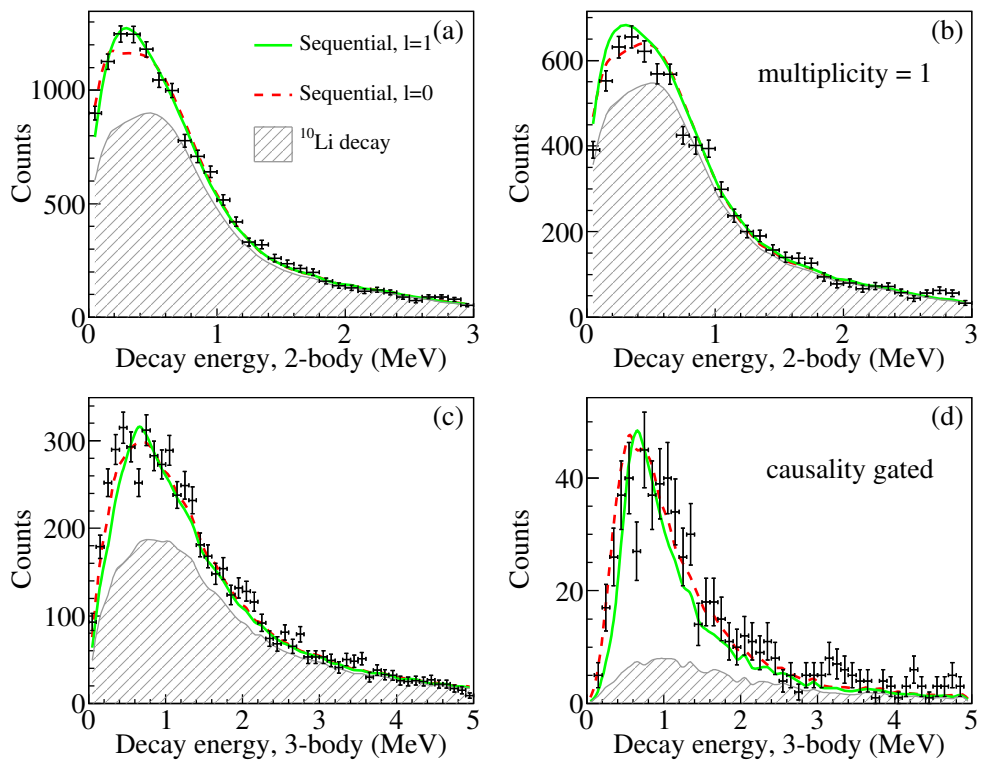

Figure 6: Two- (top row) and three-body (bottom row) decay energy spectra $\left({ }^{9} \mathrm{Li}+n\right.$ and $\left.{ }^{9} \mathrm{Li}+n+n\right)$ with fitted, simulated spectra from the two sequential decay paths. The red dashed line indicates sequential decay through the ${ }^{10} \mathrm{Li} s$-state and the green solid line indicates sequential decay through the ${ }^{10} \mathrm{Li} p$-state. The shaded regions indicate the average contribution due to the direct population of ${ }^{10} \mathrm{Li}$. 

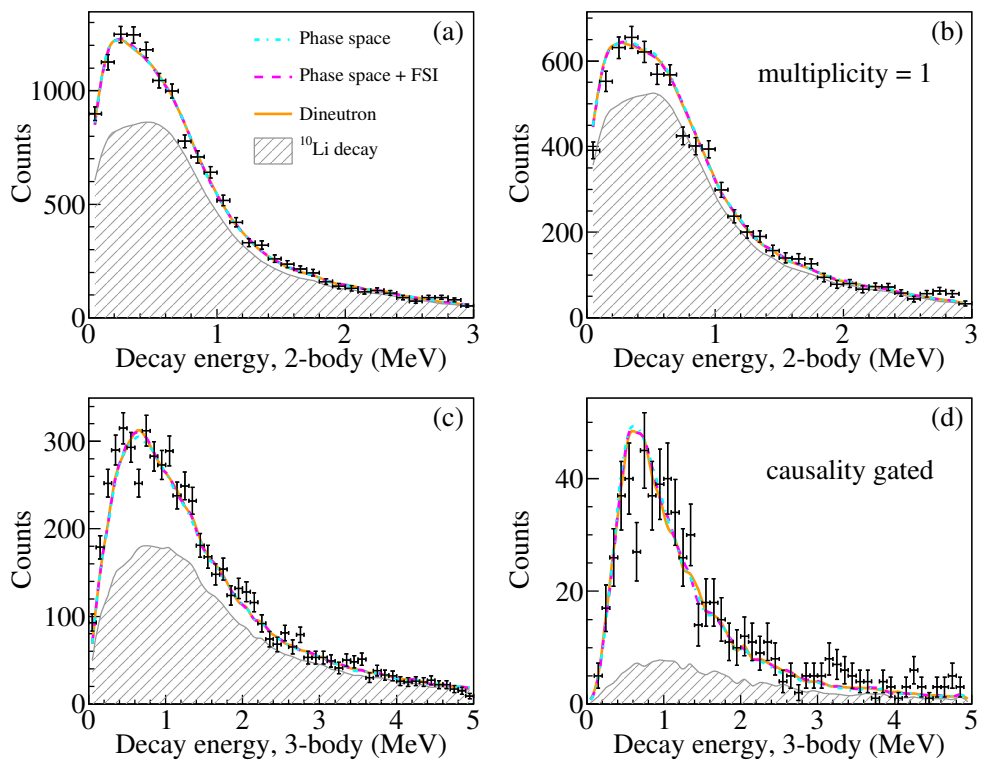

Figure 7: Two- (top row) and three-body (bottom row) decay energy spectra $\left({ }^{9} \mathrm{Li}+n\right.$ and $\left.{ }^{9} \mathrm{Li}+n+n\right)$ with fitted, simulated spectra from the three direct decay paths: phase space (cyan dot-dashed), phase space + FSI (magenta dashed), and dineutron-like (orange solid). The shaded regions indicate the average contribution due to the direct population of ${ }^{10} \mathrm{Li}$.

feature of the decay energy line shapes used in the five decay paths, an upper limit of $1300 \mathrm{keV}$ FWHM was established with an energy-independent Breit-Wigner.

The total contribution from the direct population of ${ }^{10} \mathrm{Li}$ was similar across the different decay models (maximum $20 \%$ difference in panel (a) and $<5 \%$ difference in panel (d)) and is shown as shaded areas in Figures 6 and 7 corresponding to the average sum of those contributions. The improvement in the purity of the real two-neutron events due to the causality cuts mentioned earlier is evidenced by the reduced contribution of events from the direct population of ${ }^{10} \mathrm{Li}$. These simulations show that the causality cuts removed $97 \%$ of the events incorrectly identified, due to decay of directly populated ${ }^{10} \mathrm{Li}$ or single, scattered ${ }^{11} \mathrm{Li}$ neutrons, as two-neutron events. Although they also removed approximately $40 \%$ of the true, unscattered two-neutron events, the purity of the simulated three-body decay energy spectrum (ratio of true two-neutron events to total events) was improved from $10 \%$ to $65 \%$ after causality cuts were applied.

The individual components that comprise the best fit for the dineutron model are shown in Figure 8. The quality of the fits for the other decay paths is very similar. The results of the simulations correspond to the sum (solid black line) of contributions from the direct population of the ${ }^{10} \mathrm{Li}$ ground state (dashed red line), the first excited state (dashed green line), and two high-lying states (solid grey and dot-dashed magenta lines) as well as the contribution from the population of ${ }^{11} \mathrm{Li}$ (solid orange line). In panel (b), the contribution from direct population of ${ }^{10} \mathrm{Li}$ is enhanced, while the ${ }^{11} \mathrm{Li}$ contribution is dominant in panel $(\mathrm{d})$.

While the decay energy spectra alone cannot distinguish between the five $2 n$ decay 

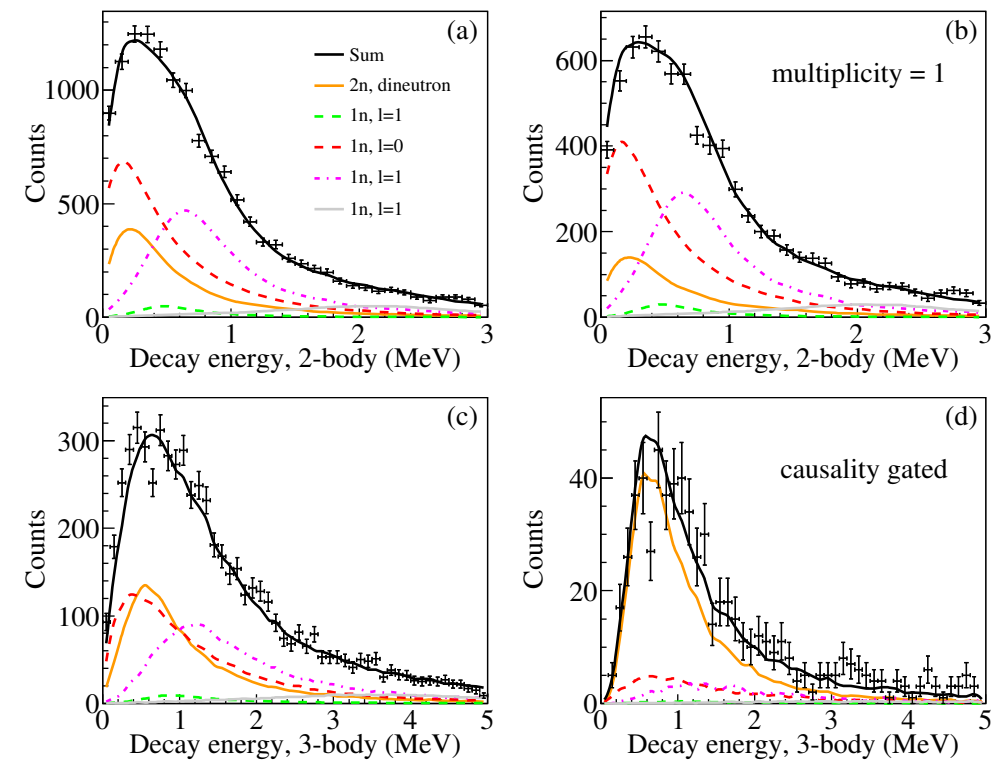

Figure 8: Two- (top row) and three-body (bottom row) decay energy spectra $\left({ }^{9} \mathrm{Li}+n\right.$ and $\left.{ }^{9} \mathrm{Li}+n+n\right)$ with fitted, simulated spectra. The data are described well with the sum (solid black line) of contributions from the direct population of the ${ }^{10} \mathrm{Li}$ ground state (dashed red line), the first excited state (dashed green line), two high-lying states (solid grey and dot-dashed magenta lines) as well as the contribution from the population of ${ }^{11} \mathrm{Li}$ (solid orange line).

\begin{tabular}{cccc}
\hline \hline & $E(\mathrm{keV})$ & $\Gamma(\mathrm{keV})$ & FWHM $(\mathrm{keV})$ \\
\hline Sequential, $\ell=1$ & $820 \pm 70$ & $>1300$ & $>400$ \\
Sequential, $\ell=0$ & $800 \pm 80$ & $>1200$ & $>850$ \\
Phase space & $720 \pm 80$ & $>1600$ & $>640$ \\
Phase space + FSI & $690 \pm 80$ & $>1700$ & $>620$ \\
Dineutron & $930 \pm 70$ & $>1800$ & $>820$ \\
\hline \hline
\end{tabular}

Table 2: Minimized ${ }^{11} \mathrm{Li}$ central energy and physical width $(\Gamma)$ values for the different $2 n$ decay paths, as well as measured FWHM values for the input total decay energy distributions. Here, $\Gamma$ refers to the intrinsic width of the state included in the calculation of the decay energy curve, while FWHM is a measurement of the width of the calculated input decay energy curve, before resolutions and acceptances are applied. As mentioned in the text, the use of energy-dependent Breit-Wigner lineshapes in these simulations only allows us to set a lower limit on the FWHM of the input distribution, but an upper limit of $1300 \mathrm{keV}$ for the FWHM was able to be set with an energy-independent Breit-Wigner. 


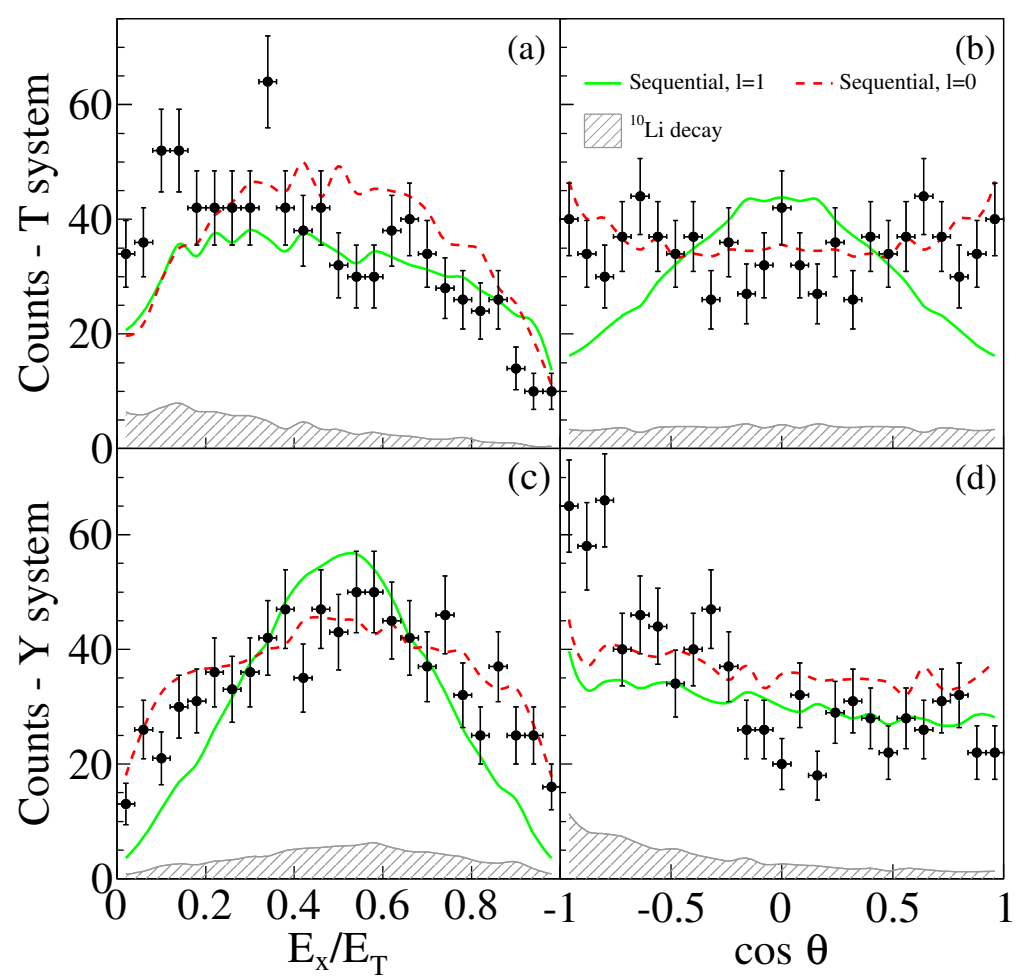

Figure 9: Jacobi plots from the decay of ${ }^{11} \mathrm{Li} \rightarrow{ }^{9} \mathrm{Li}+2 n$ events with a decay energy less than $1.6 \mathrm{MeV}$ and causality cuts applied. The lines correspond to results from the fits shown in Figure 6: sequential decay through the ${ }^{10} \mathrm{Li} s$-state (red dashed line) and sequential decay through the ${ }^{10} \mathrm{Li} p$-state (green solid line).

paths, angular correlations between the three emitted particles (two neutrons and ${ }^{9} \mathrm{Li}$ ) are expected to be more sensitive. Figure 9 shows the Jacobi plots typically employed for two-neutron [23, 24] and two-proton decays [20, 21] using the definitions of Ref. [20]. Each Jacobi system represents the three-body system as a two-body subsystem and an additional body. The two-body subsystems are neutron-neutron and core-neutron in the $T$ and $Y$ systems, respectively. The four panels in the figure display the ratio of twobody subsystem energy to three-body energy (left column) and the cosine of the angle $\theta$ between the two momentum vectors (right column) for both the $T$ (top row) and $Y$ (bottom row) systems. The data shown were gated on three-body decay energies of less than $1.6 \mathrm{MeV}$ with the causality cuts applied.

The energy ratio in the $Y$ system (Figure 10(c)) shows a symmetric distribution while the distribution of the cosine of the angle in the $T$ system (Figure 10(b)) is flat. In contrast, the energy ratio in the $T$ system (Figure 10(a)) and the cosine of the angle in the $Y$ system (Figure 10(d)) show a distinct asymmetric shape towards low energies and a strong enhancement near $\cos \theta=-1$, respectively. The latter two features indicate a tight $n-n$ subsystem with low intrinsic energy moving in the opposite direction relative to the core. 


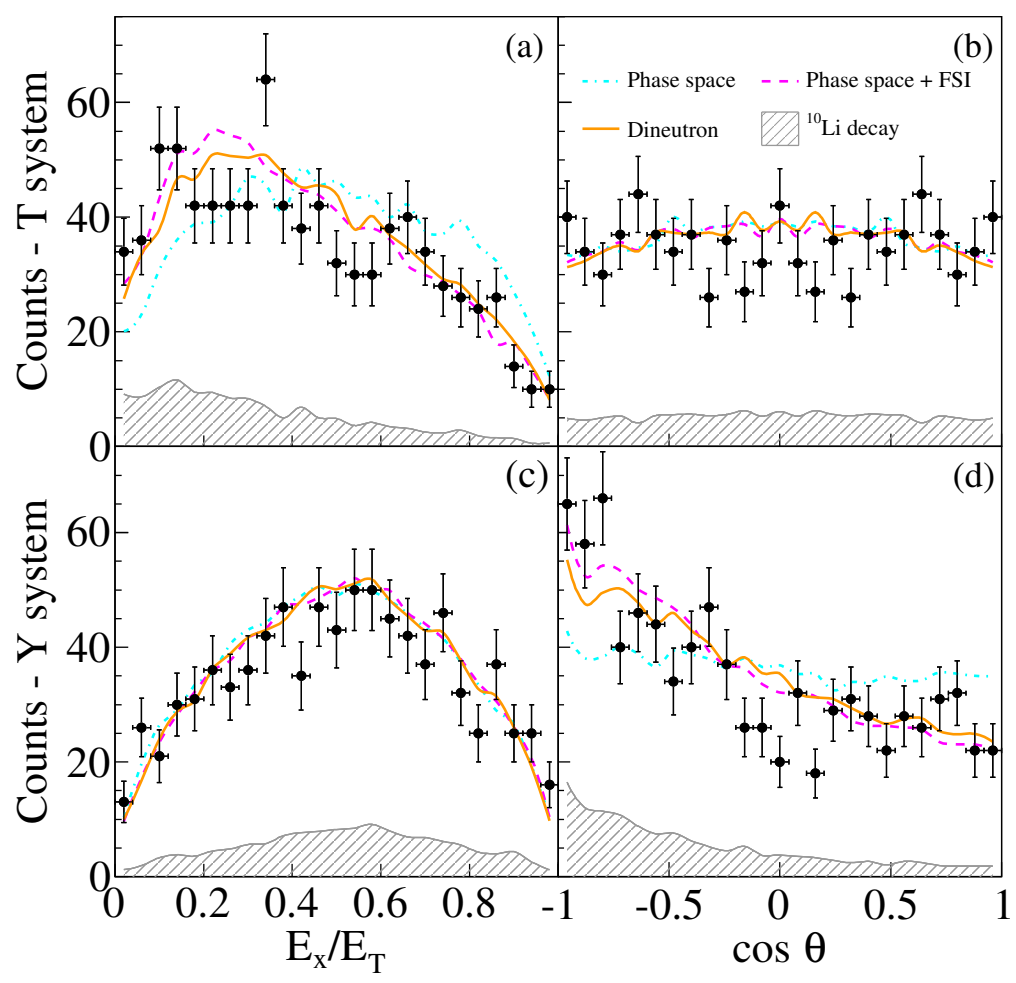

Figure 10: Jacobi plots from the decay of ${ }^{11} \mathrm{Li} \rightarrow{ }^{9} \mathrm{Li}+2 n$ events with a decay energy less than $1.6 \mathrm{MeV}$ and causality cuts applied. The lines correspond to results from the fits shown in Figure 7: phase space (cyan dot-dashed line), phase space + FSI (magenta dashed line) and dineutron (orange solid line). 
The measured Jacobi plots are compared to the minimized decay models in Figures 9 and 10, using the same energies, widths, and scaling factors that were produced by fits to the decay energy spectra. Scattered single-neutron events, such as those generated by ${ }^{10} \mathrm{Li}$ decay, have a small neutron-neutron angle, producing a peak near $\cos \theta=-1$ in the $Y$ system. The causality cuts reduce this effect significantly, but it can still be seen in the ${ }^{10} \mathrm{Li}$ contribution. In Figure 9 , the $\ell=1$ sequential decay simulation cannot reproduce the flat angular distribution in the $T$ system, while the $\ell=0$ sequential decay does a bit better. Neither sequential simulation reproduces the other three Jacobi plots.

This result is consistent with the suggestion of Bochkarev et al. that a sequential decay would not be seen unless the width of the intermediate state was much less than the energy released in the decay to that intermediate state [46]. Egorova et al. observed in their analysis of the two-proton decay of ${ }^{6} \mathrm{Be}$ that sequential decay through the intermediate states was suppressed until the total decay energy was greater than twice the intermediate state energy plus the width [21]. In the present case, the apparent width of the ${ }^{10} \mathrm{Li}$ ground state is large and the energy of the excited state is too high for a distinct sequential decay to occur.

In Figure 10, the phase space, phase space + FSI, and the dineutron models all describe the energy ratio in the $Y$ system and the $\cos \theta$ distribution in the $T$ system rather well. As expected, the phase space energy ratio distribution in the $T$ system is relatively symmetric and the $\cos \theta$ distribution in the $Y$ system is flat, neither of which agree with the asymmetry of the data. The addition of a final state interaction improves the model, as expected. Both the phase space + FSI and dineutron-like models reproduce the observed asymmetries in the $T$ system energy ratio and the $Y$ system angular distribution.

Within the uncertainty of the magnitude of the single-neutron cross-talk discussed earlier (tested with regards to the events in coincidence with ${ }^{11} \mathrm{Be}$ ), small variations in the magnitude of the cross-talk from the ${ }^{10} \mathrm{Li}$ contribution will change the relative intensities of the simulated components in the Jacobi plots. Artificially increasing the $1 n$ scattering in the spectra reduces the possible decay-path discrimination, while artificially decreasing it increases the possible discrimination. Neither variation changes the qualitative features of the spectra. The variation in the minimized energy and width of the state in ${ }^{11} \mathrm{Li}$ is small compared to the statistical and other systematic errors.

The present dineutron model is a strong simplification of a complex decay mechanism, especially for neutron unbound states which decay on a very short time scale. A full threebody model similar to the recently developed three-body cluster model used to explain correlations in the two-proton of ${ }^{6} \mathrm{Be}$ and ${ }^{16} \mathrm{Ne}$ could aid in further distinguishing this decay mechanism $[21,47]$ and with it, the central energy of this state.

\section{Summary and Conclusions}

An unbound state in ${ }^{11} \mathrm{Li}$ has been observed at a decay energy of $\sim 810 \mathrm{keV}$ corresponding to an excitation energy of $\sim 1.2 \mathrm{MeV}$. Although the decay energy spectra are insensitive to the decay model, the correlations between the ${ }^{9} \mathrm{Li}$ fragments and two neutrons are best fit by either a simple dineutron model or a phase-space plus neutronneutron FSI model. These two models suggest that the observed peak corresponds to states at $690 \pm 80 \mathrm{keV}$ or $930 \pm 70 \mathrm{keV}$, respectively, indicating that it is necessary to 
fully understand the kinematics of the $2 n$ decay before stating a central energy. A full description of the data would be assisted by fully dynamical three-body decay models.

The authors would like to thank Miguel Marqués for sharing the code for the phase space with FSI calculations with us. This work was funded by NSF grant nos. PHY096173, PHY-1102511, PHY-1205537, and PHY-1306074 and DOE Grant No. DE-SC0009883. This material is based upon work supported by the Department of Energy National Nuclear Security Administration under Grant No. DE-NA0000979.

\section{References}

[1] K. Riisager, Halos and related structures, Physica Scripta T152 (2013) 014001.

[2] T. Aumann, T. Nakamura, The electric dipole response of exotic nuclei, Physica Scripta T152 (2013) 014012 .

[3] H. Simon, Halo nuclei, stepping stones across the drip-lines, Physica Scripta T152 (2013) 014024.

[4] F. M. Marqués, et al., Two-neutron interferometry as a probe of the nuclear halo, Physics Letters B 476 (2000) 219-225.

[5] I. Tanihata, H. Savajols, R. Kanungo, Recent experimental progress in nuclear halo structure studies, Progress in Particle and Nuclear Physics 68 (2013) 215-313.

[6] T. Kobayashi, Projectile fragmentation of exotic nuclear beams, Nuclear Physics A 538 (1992) 343-352.

[7] D. Sackett, et al., Electromagnetic excitation of ${ }^{11} \mathrm{Li}$, Physical Review C 48 (1993) 118-135.

[8] K. Ieki, et al., Coulomb dissociation of ${ }^{11} \mathrm{Li}$, Physical Review Letters 70 (1993) 730-733.

[9] S. Shimoura, T. Nakamura, M. Ishihara, N. Inabe, T. Kobayashi, T. Kubo, R. Siemssen, I. Tanihata, Y. Watanabe, Coulomb dissociation reaction and correlations of two halo neutrons in ${ }^{11} \mathrm{Li}$, Physics Letters B 348 (1995) 29-34.

[10] M. Zinser, et al., Invariant-mass spectroscopy of ${ }^{10} \mathrm{Li}$ and ${ }^{11} \mathrm{Li}$, Nuclear Physics A 619 (1997) $151-176$.

[11] H. Simon, et al., Systematic investigation of the drip-line nuclei ${ }^{11} \mathrm{Li}$ and ${ }^{14} \mathrm{Be}$ and their unbound subsystems ${ }^{10} \mathrm{Li}$ and ${ }^{13} \mathrm{Be}$, Nuclear Physics A 791 (2007) 267-302.

[12] M. G. Gornov, Y. Gurov, S. Lapushkin, P. Morokhov, V. Pechkurov, T. K. Pedlar, K. K. Seth, J. Wise, D. Zhao, Excited States of ${ }^{11} \mathrm{Li}$, Physical Review Letters 81 (1998) 4325-4328.

[13] T. Nakamura, et al., Observation of Strong Low-Lying E1 Strength in the Two-Neutron Halo Nucleus ${ }^{11} \mathrm{Li}$, Physical Review Letters 96 (2006) 252502.

[14] M. Smith, et al., First Penning-Trap Mass Measurement of the Exotic Halo Nucleus ${ }^{11}$ Li, Physical Review Letters 101 (2008).

[15] T. Kobayashi, Nuclear structure experiments on ${ }^{11} \mathrm{Li}$, Nuclear Physics A 553 (1993) 465-472.

[16] A. A. Korsheninnikov, et al., Spectroscopy of the halo nucleus ${ }^{11} \mathrm{Li}$ by an experimental study of ${ }^{11} \mathrm{Li}+p$ collisions, Physical Review C 53 (1996) R537-R540.

[17] A. A. Korsheninnikov, et al., $L=1$ Excitation in the Halo Nucleus ${ }^{11}$ Li, Physical Review Letters 78 (1997) 2317-2320.

[18] R. Kanungo, et al., Evidence of Soft Dipole Resonance in ${ }^{11}$ Li with Isoscalar Character, Physical Review Letters 114 (2015) 192502.

[19] H. Johansson, et al., Three-body correlations in the decay of $10 \mathrm{He}$ and 13Li, Nuclear Physics A 847 (2010) 66-88.

[20] R. J. Charity, et al., Investigations of three-, four-, and five-particle decay channels of levels in light nuclei created using a ${ }^{9} \mathrm{C}$ beam, Physical Review C 84 (2011) 014320.

[21] I. A. Egorova, et al., Democratic Decay of ${ }^{6}$ Be Exposed by Correlations, Physical Review Letters 109 (2012) 202502.

[22] A. Spyrou, et al., First Observation of Ground State Dineutron Decay: ${ }^{16}$ Be, Physical Review Letters 108 (2012) 102501.

[23] Z. Kohley, et al., First observation of the ${ }^{13}$ Li ground state, Physical Review C 87 (2013) 011304.

[24] Y. Aksyutina, et al., Study of the ${ }^{14}$ Be Continuum: Identification and Structure of its Second $2^{+}$ State, Physical Review Letters 111 (2013) 242501.

[25] M. D. Jones, et al., Two-neutron sequential decay of ${ }^{24}$ O, Physical Review C 92 (2015) 051306.

[26] F. Marti, Commissioning of the Coupled Cyclotron system at NSCL, in: AIP Conference Proceedings, volume 600, AIP, 2001, pp. 64-68. URL: http://scitation.aip.org/content/aip/ proceeding/aipcp/10.1063/1.1435199. doi:10.1063/1.1435199. 
[27] D. J. Morrissey, B. M. Sherrill, M. Steiner, A. Stolz, I. Wiedenhoever, Commissioning the A1900 projectile fragment separator, Nuclear Instruments and Methods in Physics Research Section B: Beam Interactions with Materials and Atoms 204 (2003) 90-96.

[28] M. D. Bird, S. J. Kenney, J. Toth, H. W. Weijers, J. C. DeKamp, M. Thoennessen, A. F. Zeller, System Testing and Installation of the NHMFL/NSCL Sweeper Magnet, IEEE Transactions on Appiled Superconductivity 15 (2005) 1252-1254.

[29] B. Luther, et al., MoNAThe Modular Neutron Array, Nuclear Instruments and Methods in Physics Research Section A: Accelerators, Spectrometers, Detectors and Associated Equipment 505 (2003) $33-35$.

[30] J. K. Smith, et al., Low-lying neutron unbound states in ${ }^{12}$ Be, Physical Review C 90 (2014) 024309.

[31] J. K. Smith, Unbound States in the Lightest Island of Inversion: Neutron Decay Measurements of ${ }^{11} \mathrm{Li},{ }^{10} \mathrm{Li}$, and ${ }^{12} \mathrm{Be}$, Ph.D. thesis, Michigan State University, 2014. URL: https://publications . nscl.msu.edu/thesis/Smith2014_372.pdf.

[32] J. Wang, A. Galonsky, J. Kruse, P. Zecher, F. Deák, A. Horváth, A. Kiss, Z. Seres, K. Ieki, Y. Iwata, Neutron cross-talk in a multi-detector system, Nuclear Instruments and Methods in Physics Research Section A: Accelerators, Spectrometers, Detectors and Associated Equipment 397 (1997) 380-390.

[33] F. Marqués, M. Labiche, N. Orr, F. Sarazin, J. C. Angélique, Neutron cross-talk rejection in a modular array and the detection of halo neutrons, Nuclear Instruments and Methods in Physics Research Section A: Accelerators, Spectrometers, Detectors and Associated Equipment 450 (2000) $109-118$.

[34] Z. Kohley, et al., Modeling interactions of intermediate-energy neutrons in a plastic scintillator array with Geant4, Nuclear Instruments and Methods in Physics Research Section A: Accelerators, Spectrometers, Detectors and Associated Equipment 682 (2012) 59-65.

[35] S. Agostinelli, et al., Geant4 - a simulation toolkit, Nuclear Instruments and Methods in Physics Research Section A: Accelerators, Spectrometers, Detectors and Associated Equipment 506 (2003) 250-303.

[36] J. Allison, et al., Geant4 developments and applications, IEEE Transactions on Nuclear Science 53 (2006) 270-278.

[37] B. R. Marks, et al., Population of ${ }^{13}$ Be in a nucleon exchange reaction, Physical Review C 92 (2015) 054320

[38] A. Volya, Physics of unstable nuclei: from structure to sequential decays, EPJ Web of Conferences 38 (2012) 03003

[39] F. E. James, Monte Carlo phase space, in: Academic Training Lectures, CERN, Geneva, Switzerland, 1968, p. 41. URL: http://cds.cern.ch/record/275743. doi:10.5170/CERN-1968-015.

[40] F. Marqués, et al., Three-body correlations in Borromean halo nuclei, Physical Review C 64 (2001) 061301.

[41] D. Gonzalez Trotter, et al., Neutron-deuteron breakup experiment at $E_{n}=13 \mathrm{MeV}$ : Determination of the ${ }^{1} S_{0}$ neutron-neutron scattering length $a_{n n}$, Physical Review C 73 (2006) 034001.

[42] M. Wang, G. Audi, A. Wapstra, F. Kondev, M. MacCormick, X. Xu, B. Pfeiffer, The Ame2012 atomic mass evaluation, Chinese Physics C 36 (2012) 1603-2014.

[43] H. G. Bohlen, A. Blazevic, B. Gebauer, W. Von Oertzen, S. Thummerer, R. Kalpakchieva, S. M. Grimes, T. N. Massey, Spectroscopy of exotic nuclei with multi-nucleon transfer reactions, Progress in Particle and Nuclear Physics 42 (1999) 17-26.

[44] P. Santi, et al., Structure of the ${ }^{10} \mathrm{Li}$ nucleus investigated via the ${ }^{9} \mathrm{Li}(\mathrm{d}, \mathrm{p}){ }^{10} \mathrm{Li}$ reaction, Physical Review C 67 (2003) 024606.

[45] J. Smith, et al., Selective population of unbound states in ${ }^{10} \mathrm{Li}$, Nuclear Physics A 940 (2015) $235-241$.

[46] O. V. Bochkarev, L. V. Chulkov, A. A. Korsheninnikov, E. A. Kuz'min, I. G. Mukha, G. B. Yankov, Democratic decay of ${ }^{6}$ Be states, Nuclear Physics A 505 (1989) 215-240.

[47] K. W. Brown, et al., Interplay between sequential and prompt two-proton decay from the first excited state of ${ }^{16} \mathrm{Ne}$, Physical Review C 92 (2015) 034329. 